\title{
ESTUDO PROSPECTIVO E RANDOMIZADO DE PACIENTES COM FRATURAS EXPOSTAS DA DIÁFISE DO FÊMUR SUBMETIDOS A OSTEOSSÍNTESE COM PLACA E HASTE INTRAMEDULAR BLOQUEADA A FOCO ABERTO
}

\author{
PROSPECTIVE AND RANDOMIZED STUDY OF PATIENTS WITH OPEN FRACTURES OF THE FEMORAL SHAFT, \\ TREATED WITH PLATE OR OPEN INTRAMEDULLARY LOCKED NAIL
}

\author{
antonio Marcos Ferracini ${ }^{1}$, Flavio Faloppa², Gildasio de Cerqueira Daltro 3 , Dalton de Castro Crisóstomo Júnior 4 , \\ FeRnando Baldy dos ReIS ${ }^{5}$, JoÃo Carlos Belotti ${ }^{6}$
}

\begin{abstract}
RESUMO
Trata-se de um estudo prospectivo e randomizado de duas técnicas de osteossíntese no tratamento das fraturas expostas diafisárias do fêmur, realizado entre janeiro de 2002 a abril de 2004. Haste intramedular bloqueada fresada realizada a foco aberto e placa e parafusos foram empregadas no tratamento de 20 pacientes em cada grupo. De acordo com a classificação de Gustilo, 26 (65\%) foram tipo I, 10 (25\%) tipo II e 4 (10\%) tipo IIIA. Quanto ao mecanismo das fraturas, 21 por trauma contuso e 19 por ferimentos de arma de fogo.Três pacientes foram excluídos nas avaliação final. Houve complicação em 12 (32,4\%), sendo 4 no grupo de placas e 8 no grupo das hastes. $O$ grupo de haste bloqueada apresentou $2(10 \%)$ infecções profundas, 2 infecções superficiais (10\%), 1 falha de consolidação (5\%). O grupo de placa e parafusos resultou em 1 infecção profunda associada à falha de consolidação (5,8\%), 1 infecção superficial (5,8\%). Pela classificação de resultados de Thorensen obteve-se bons e excelentes resultados em 28 $(75,7 \%)$ fraturas, $3(7.5 \%)$ casos regulares e $6(15 \%)$ casos ruins. A estabilização com placas e parafusos, trouxe menores taxas de complicações, quando comparadas com o uso de hastes fresadas a foco aberto,embora sem significado estatístico.
\end{abstract}

Descritores: Fixação intramedular de fraturas; Fraturas do fêmur; Placas ósseas; Diáfises; Fraturas expostas.

Citação: Ferracini AM, Faloppa F, Daltro GC, Crisóstomo DC Jr, Reis FB, Belotti JC.Estudo prospectivo e randomizado de pacientes com fraturas expostas da diáfise do fêmur submetidos a osteossíntese com placa e haste intramedular bloqueada a foco aberto. Acta Ortop Bras. [periódico na Internet]. 2008; 16(2):102-106. Disponível em URL: http://www.scielo.br/aob.

\section{INTRODUÇÃO}

As vantagens e desvantagens da haste bloqueada utilizada para a estabilização das fraturas expostas da diáfise do fêmur, raramente são comparadas à estabilização com placa, não obstante ser a técnica mais apresentada nas publicações.

A abordagem dessa lesão apresenta uniformidade de conduta sobre a necessidade de receber desbridamento cirúrgico de ur-

\section{SUMMARY}

We assessed the complications and treatment outcomes in a prospective and randomized study of two osteosynthesis techniques in open femoral shaft fractures. Between January 2002 and April 2004, 40 patients with open fractures of the femoral shaft were assessed, with 20 being treated with open reamed intramedullary locked nail and 20 with plate. Twenty-six (65\%) fractures were classified as Gustilo type -I open fractures; ten (25\%) as type II and four (10\%), as type IIIA. There were 21 blunt injuries and 19 gunshot injuries. Three patients were excluded from the final assessment. Complications were observed in 12 (32.4\%) patients, 4 in the plate group and 8 in the nail group. Reamed intramedullary locked nail resulted in two deep infections (10\%), two superficial infections (10\%), and one nonunion (5\%). With plate techniques, we had one deep infection associated to nonunion $(5,8 \%)$ and one superficial infection (5.8\%). Good and excellent outcomes were found in 28 fractures $(75.7 \%)$, fair $(7.5 \%)$ in three cases, and poor $(15 \%)$ in six, according to Thorensen's criteria. In this study, the stabilization with plate results in lower complications rate when compared to the open intramedullary nail, although with no statistical significance.

Keywords: Fracture fixation intramedullary; Femoral fractures; Bone plates; Diaphyses; Fractures open.

Citation: Ferracini AM, Faloppa F, Daltro GC, Crisóstomo DC Jr, Reis FB, Belotti $J C$. Prospective and randomized study of patients with open fractures of the femoral shaft, treated with plate or open intramedullary locked nail. Acta Ortop Bras. [serial on the Internet]. 2008; 16(2):102-106. Available from URL: http://www.scielo.br/aob.

gência e estabilização imediata sempre que possível(1). Entretanto, a escolha da síntese e da técnica de aplicação em nosso meio, suscita alguma discussão e não raramente as estabilizações são diferidas e realizadas com placas.

As placas como forma de fixação das fraturas expostas da diáfise do fêmur são realizadas com alguma reserva, devido a receios que surgiram com os altos índices de complicações observados quando a técnica era empregada visando redução anatômica e

Trabalho realizado no Curso de Pós Graduação em Ortopedia e Traumatologia da Universidade Federal de São Paulo - UNIFESP/EPM e no Serviço de Ortopedia e Traumatologia, Faculdade de Medicina, Hospital Universitário Professor Edgar Santos - HUPES - UFBA.

Endereço para correspondência: PG em Ortopedia: Rua Borges Lagoa, 783 - 5o andar-São Paulo, Brasil.CEP: 04038-002 .E-mail: fbaldy@superig.com.br

1. Professor Auxiliar de Ortopedia e Traumatologia da Faculdade de Medicina da Universidade Federal da Bahia - UFBA.

2. Professor Titular do Departamento de Ortopedia e Traumatologia do Departamento de Ortopedia e Traumatologia Universidade Federal de São Paulo - UNIFESP - Escola Paulista de Medicina - EPM.

3. Professor livre Docente da Faculdade de Medicina, Chefe do Serviço de Ortopedia do Hospital Universitário Professor Edgard Santos - HUPES.

4. Médico do Serviço de Ortopedia e Traumatologia do HUPES.

5. Professor Associado e Livre Docente da Disciplina de Traumatologia do Departamento de Ortopedia e Traumatologia Universidade Federal de São Paulo - UNIFESP - Escola Paulista de Medicina - EPM

6. Médico Assistente Doutor do Departamento de Ortopedia e Traumatologia Universidade Federal de São Paulo - UNIFESP - Escola Paulista de Medicina - EPM. 
compressão interfragmentária. A revisão dos conceitos de síntese com a aplicação de princípios para a proteção das partes moles, revitalizaram sua utilização(2).

Embora haja alguma controvérsia em relação ao uso de hastes intramedulares no que diz respeito à técnica de aplicação, fresada ou não e também em função das possíveis complicações locais e sistêmicas, essa é uma forma de estabilização muito utilizada e os resultados apresentados na literatura para as fraturas expostas da diáfise do fêmur, são muito favoráveis $(3,4)$.

Objetivando identificar os resultados do tratamento, as complicações e sua freqüência nos pacientes com fraturas expostas diafisárias do fêmur em nosso serviço, realizamos um estudo comparativo prospectivo e randomizado das duas técnicas, com um grupo tratado com placas e parafusos e outro com haste intramedular bloqueada fresada em foco aberto.

\section{MATERIAL E MÉTODOS}

Foi realizado um ensaio clínico randomizado de 40 pacientes portadores de fraturas expostas da diáfise do fêmur, submetidos a desbridamento de urgência e antibioticoterapia conforme protocolo de Gustilo et al. ${ }^{(5)}$.Após a limpeza inicial permaneceram em tração esquelética para estabilização em um segundo tempo.Como fatores de inclusão,estudamos apenas as fraturas expostas diafisárias do fêmur, em esqueleto maduro e com limpeza cirúrgica realizada dentro de seis horas do trauma e sem complicações sistêmicas. O paciente era convidado a participar do estudo a partir de uma explicação do projeto da pesquisa e aceitando, assinava o termo de consentimento informado. Após randomização por envelopes lacrados, 20 pacientes foram submetidos a osteossíntese com placa e parafusos e 20 com haste intramedular bloqueada e fresada realizada a foco aberto. As cirurgias foram realizadas em Hospital da rede pública no período de janeiro de 2002 a abril de 2004. Os pacientes foram acompanhados por período de nove meses quando eram considerados curados ou com falha de consolidação necessitando nova abordagem.

As fraturas, quanto ao mecanismo, foram classificadas em trauma contuso e penetrante. O tipo de exposição foi classificado de acordo com Gustilo, quanto à configuração da fratura utilizamos a classificação da $\mathrm{AO}^{(6)}$.

Retarde de consolidação foi considerado para a fratura não consolidada até a $26^{a}$ semana, e falha de consolidação após nove meses sem evolução radiográfica ${ }^{(7)}$.

Foi empregado o método de Thoresen et al. ${ }^{(8)}$, para a avaliação clínica dos resultados.

\section{Técnicas operatórias}

Haste bloqueada - Os pacientes foram operados em mesa cirúrgica padrão e em decúbito lateral com acesso posterior e abertura do foco de fratura para auxiliar a passagem do fio guia. Utilizou-se uma haste de fabricação nacional, rígida, com leve curvatura anterior, canulada e cilíndrica, sendo realizado redução manual e fresagem do canal para a introdução da haste de maneira anterógrada. Possuía um gabarito para inserção dos parafusos de bloqueio distal e proximal, ambos estáticos.

Placa e parafuso - Os pacientes foram operados em mesa padrão. A abordagem foi realizada com a menor agressão às partes moles quando possível, sendo inclusive, em alguns casos, utilizada a técnica de placa ponte de maneira formal com duas pequenas incisões laterais. A escolha do material de síntese e o grau de satisfação com o resultado ficaram a cargo do cirurgião responsável. Fixou-se a placa com um mínimo de oito corticais em cada lado da fratura.

\section{Análise estatística}

Para a descrição das variáveis contínuas, usou-se a média com o desvio padrão e a mediana. Para testar as variáveis contínuas, foi utilizado o teste $t$ de Student ou o teste de Mann-Whitney. Para comparação de três ou mais grupos foi usada a análise de variância (ANOVA).

Na comparação de variáveis categóricas se usou teste de qui-quadrado ou teste Exato de Fisher. O teste de Kolmogorov-Smirnov foi usado para avaliar se as variáveis contínuas possuíam uma distribuição semelhante à distribuição normal. Os grupos de pacientes foram comparados com o erro alfa de $5 \%$ com significância para $\mathrm{p}<0,05$.

\section{RESULTADOS}

As características gerais dos grupos estudados são observadas na Tabela 1.

Em três casos de pacientes com fraturas de fêmur provocadas por projéteis de arma de fogo (casos 8, 15 e 17), houve quebra da randomização, pois faltava ao cirurgião experiência com a inserção da haste intramedular bloqueada e os pacientes receberam tratamento com placa e parafuso. Esses casos foram excluídos da casuística, restando então 37 casos.

Lesões associadas foram vistas em 12 pacientes $(32,4 \%-n=37)$ no estudo: dois pacientes com TCE, duas lesões neurológicas

\begin{tabular}{l|l|l|l|l}
\hline \multirow{2}{*}{ Características } & & & \\
\cline { 2 - 4 } & & & & Haste \\
\hline Sexo & $\mathrm{M}$ & $15(88,2 \%)$ & $19(95,0 \%)$ & $0,452^{*}$ \\
\hline Idade(anos) & $\mathrm{F}$ & $2(11,8 \%)$ & $1(5,0 \%)$ & \\
\hline Espera para síntese & Média $\pm \mathrm{dp}$ & & $27,65 \pm 8,4$ & $0,75 \dagger$ \\
\hline Lado acometido & Média \pm dp & & $14,05 \pm 8,7$ & $0,209 \dagger$ \\
\hline & $\mathrm{D}$ & $8(47,1 \%)$ & $12(60,0 \%)$ & $0,43^{*}$ \\
\hline Mecanismo da lesão & $\mathrm{E}$ & $9(52,9 \%)$ & $8(40,0 \%)$ & \\
\hline & Penetrante & $11(64,7 \%)$ & $5(25,0 \%)$ & $0,015^{*}$ \\
\hline
\end{tabular}

$M=$ masculino; F= feminino / $D=$ direito; $E=$ esquerdo / - Média $\pm d p=$ média \pm desvio padrão / - *Teste do X / - †Teste Mann-Whitney / Mann-Whitney test. - Fonte (Source): Serviço de ortopedia e traumatologia do HUPES.

Tabela 1- Características gerais (sexo, idade, dias de espera para osteossíntese, lado acometido e mecanismo da lesão) 
periféricas, uma lesão cardíaca por projétil de arma de fogo, duas fraturas de tíbia, uma fratura de fêmur contra-lateral, uma fratura de tornozelo, uma fratura do anel pélvico, uma fratura de diáfise de úmero e uma fratura-luxação do joelho.

Com relação ao mecanismo da lesão, houve 11 lesões penetrantes e 6 contusas no grupo da placa e 5 lesões penetrantes e 15 contusas no grupo das hastes. A Tabela 2 apresenta as fraturas com os respectivos tratamentos de acordo com a classificação de Gustilo.Com relação à configuração das fraturas, segundo a classificação da AO, encontrou-se $59,4 \%$ do tipo B, $29,7 \%$ do tipo A e $10,8 \%$ do tipo C.

\begin{tabular}{l|l|l|l|l}
\hline & & \multicolumn{2}{|l|}{ Síntese utilizada } & Total \\
\hline & & Placa № (\%) & Haste № (\%) & № (\%) \\
\hline \multirow{2}{*}{$\begin{array}{l}\text { Classificação } \\
\text { de Gustilo }\end{array}$} & I & $13(76,5)$ & $10(50)$ & $23(62,2)$ \\
\cline { 2 - 5 } & IIIA & $2(11,8)$ & $2(10)$ & $\mathbf{4}(10,8)$ \\
\cline { 2 - 5 } & & $17(100)$ & $20(100)$ & $\mathbf{3 7 ( 1 0 0 )}$ \\
\hline Total (No) & & $8(40)$ & 10 \\
\hline
\end{tabular}

Teste exato de Fisher $/$ Fisher's exact test = 0,159 - Fonte: Serviço de ortopedia e traumatologia do HUPES

Tabela 2 - Distribuição dos pacientes de acordo com o tipo de tratamento e a classificação de Gustilo

As complicações foram observadas em 12 pacientes $(32,4 \%$ - $n=37)$, sendo 4 no grupo de placas $(23,5 \%)$ e 8 no grupo das hastes $(40,0 \%), p=0,286$. (Gráfico 1) Usando a classificação de Gustilo, para o tipo I houve complicações em $26,1 \%(n=23)$, no tipo II 40,0\% ( $n=10)$ e no tipo III $50 \%(n=4)$, tendo um $p=0,51$. Usando a classificação da $\mathrm{AO}$, para o tipo A houve complicações em $9,1 \%(n=11)$, no tipo B 40,9\% $(n=22)$ e no tipo C $50 \%$ $(n=4)$, tendo um $p=0,90$. Com relação à infecção encontrou-se no grupo das hastes dois pacientes com infecções profundas (10\%) e dois com infecções superficiais (10\%) e no grupo de placas, uma infecção profunda (5,8\%) e uma superficial $(5,8 \%)$. Falha de consolidação ocorreu em um paciente do grupo das placas e esteve associado à infecção profunda e em um paciente do grupo de hastes associado à falha técnica. Dois pacientes no grupo de hastes e um paciente no grupo de placas tiveram encurtamentos significativos, maior que dois centímetros. Um desvio angular maior que dez graus foi encontrado em um paciente de cada grupo (Tabela 3). Todos os casos de infecção estiveram associados a algum grau de rigidez articular, mas nenhum com menos 90 graus de flexão.

Não foram encontradas complicações sistêmicas nos pacientes estudados.

A consolidação ocorreu em uma média geral de 20,71 2 2,72 semanas, sendo 21,38 $\pm 3,07$ (16 pacientes sem o caso de infecção profunda) semanas para as fraturas estabilizadas com placa e parafusos e 20,07 semanas (17 pacientes, sem os casos de infecção profunda e falha de consolidação) para as fraturas estabilizadas com haste bloqueada. Houve um paciente do grupo das placas, que consolidou com 26,7 semanas, sendo considerado um caso de retarde de consolidação e esteve associado à infecção superficial. Na avaliação do tempo de consolidação de acordo com a classificação de Gustilo, encontramos para o tipo I uma média de $20,29 \pm 2,9$, tipo II 21,7 $\pm 2,2$ e tipo III 21,62 $\pm 2,2(p=0,425)$. Para o tempo de consolidação, segundo a classificação da AO,

o tipo A teve média de 19,97 $\pm 2,29$, tipo B 21,42 \pm 2,91 e tipo C $19,55 \pm 2,65(p=0,259)$.

Pela classificação de Thoresen obteve-se excelentes e bons resultados em 28 fraturas $(75,7 \%)$. Três casos $(8,1 \%)$ foram considerados regulares e decorrentes das infecções superficiais acompanhados de rigidez articular. Seis casos ruins (16,2\%) representando as três infecções profundas, um caso de falta de consolidação associado à falha do material e dois encurtamentos significativos. Na análise dos grupos foi encontrado $82,4 \%(n=17)$ de excelentes e bons resultados para o grupo das placas e $70 \%(n=20)$ para o grupo das hastes, $p=0,84$.

\section{DISCUSSÃO}

As fraturas expostas da diáfise do fêmur são lesões graves que pressupõem maior taxa de complicações no tratamento podendo variar de 0 a 24\%(3,9-11). Embora nossa série seja composta de tipos de exposição óssea que prenunciam bons resultados, tipo I, II e IIIA, encontramos complicações gerais em 32,4\%, um índice muito elevado, mas próximo ao apresentado por outros autores ${ }^{(1)}$. As lesões penetrantes, decorrentes de ferimentos por projéteis de arma de fogo de baixa energia, foram incluídas nesta avaliação por terem sido abordadas na emergência com desbridamento e limpeza do foco de fratura. Para esse grupo obtivemos uma menor quantidade de complicações e melhores resultados pela classificação de Thoresen o que coincide com a literatura(12,13). (Figuras 1 e 2).

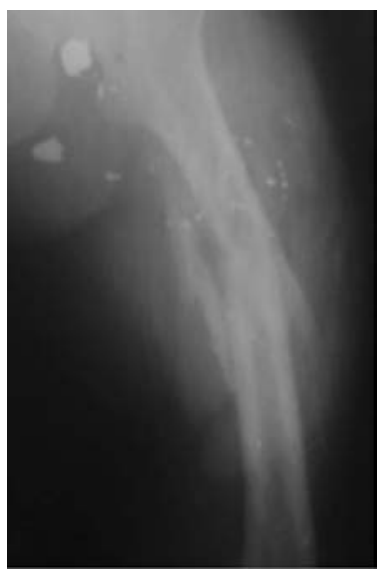

Figura 1 - 20 anos, masculino. Fratura exposta do fêmur $E$ por ferimento de arma de fogo classificada como Gustilo tipo I , AO-B2.

O momento de fixar as fraturas expostas já foi motivo de discussão, mas a conduta atual é estabilizar no ato do desbridamento, desde que realizado em tempo hábil e haja cobertura da estrutura óssea ${ }^{(14)}$. Os pacientes deste estudo aguardaram uma média de 11,4 8,44 dias até a estabilização definitiva, tempo esse considerado longo quando comparado com outras séries ${ }^{(15,16)}$. Esse período de espera não foi intencional, mas devido à grande demanda do serviço público. Na avaliação das complicações constatou-se que os pacientes que complicaram tiveram uma média maior de espera, 16,75 \pm 9,93 dias, contra os que não complicaram, 9,68 $\pm 6,86$ dias e esta diferença foi estatisticamente significante, $p=$ 0,039(teste $t$ de Student). Isto em parte, pode ser explicado pela dificuldade em reduzir a fratura de maneira adequada.

Usando a classificação da AO para avaliar se o tipo da fratura influenciava na média do tempo de consolidação e no índice com- 
plicações, não foi observada diferença estatística significante $(p=$ 0,26 , teste de ANOVA).

A Tabela 3 mostra uma maior proporção de complicações para a classificação de Gustilo III-A, mas não foi estatísticamente significante, $p=038$.

A infecção, condição mais temida das fraturas expostas pode variar no tratamento de 0 a 16\%(1,3,9,10,15). Riemer et al. ${ }^{(17)}$ discutiram os resultados de 141 fraturas do fêmur tratadas com placas, entre elas 49 fraturas expostas com $2 \%$ de infecção. O'Brien et al. ${ }^{(1)}$ estudaram 63 fraturas expostas do fêmur tratadas de forma primária com haste intramedular bloqueada fresada com 12\% de infecção. A taxa global de infecção foi de 16,2\%, com 20\% para o grupo da haste e11,6\% para o grupo das placas sendo considerados altos quando comparados com a literatura $(1,15,18)$. Em toda série encontramos infecção profunda em três pacientes $(8,1 \%)$ e superficial em três $(8,1 \%)$. No grupo das hastes houve duas infecções profundas (10\%) e duas superficiais (10\%) e no grupo de placas, uma infecção profunda $(5,8 \%)$ e uma superficial (5,8\%). O paciente com infecção profunda no grupo de placas, evoluiu para falha de consolidação, necessitando de aplicação da técnica de transporte ósseo. Os dois pacientes do grupo da haste receberam novos desbridamentos e antibioticoterapia, evoluindo para consolidação, embora, com limitação da flexão do joelho em $90^{\circ}$. Após a cura do processo infeccioso as hastes foram retiradas. As infecções superficiais, em número de três, foram tratadas de maneira definitiva com antibioticoterapia.

Falha de consolidação foi encontrada em dois pacientes $(5,4 \%)$ : um paciente tratada com placa e um paciente tratado com haste. Uma fratura em retarde de consolidação evoluiu para cura após o tratamento da infecção superficial. Esses índices são semelhantes aos encontrados na literatura ${ }^{(1,3,11,15)}$.

Três pacientes finalizaram o tratamento com encurtamento, um no grupo de placas e dois no grupo das hastes. Os desvios angulares, em número de dois, foram de 10 graus, um valgo para o grupo das hastes e um varo para o grupo das placas, observado também em outras séries ${ }^{(15)}$.

Comparando as duas formas de estabilização, observa-se que a incidência de complicações locais foi maior para as fraturas tratadas com hastes (Gráfico 1), entretanto essa diferença não é estatisticamente significante ( $p=0,168)$.

Não houve diferença com significado estatístico no tempo de consolidação entre os dois grupos.

As complicações aqui discutidas aproximam-se dos resultados publicadas para as fraturas fechadas tratadas com placas ${ }^{(17)}$, mas podem ser considerados números elevados para os pacientes tra-

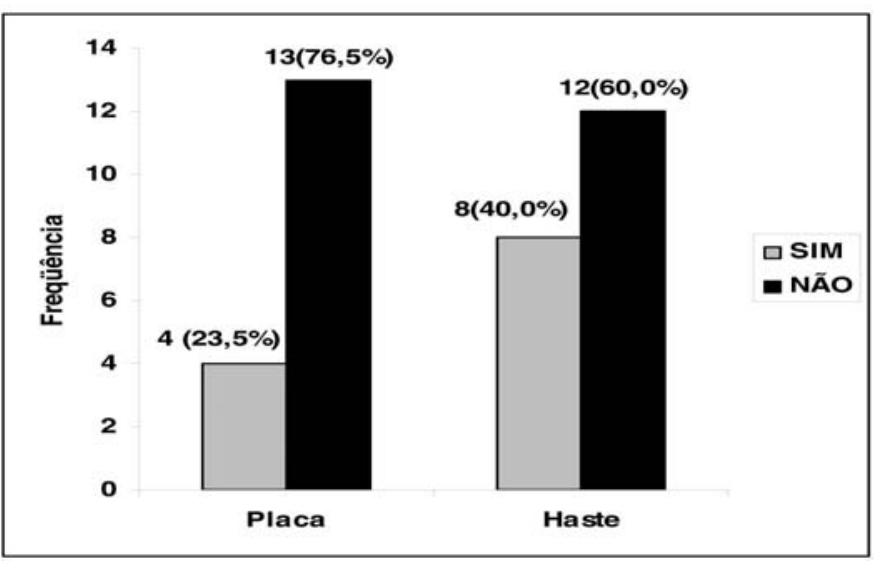

Gráfico 1 - Freqüência de complicações por tipo de síntese $(p=0,319)$.

\begin{tabular}{|c|c|c|c|c|c|c|}
\hline Caso & Mec.lesão & Class.Gustilo & Class.AO & T.espera & Sínt. & Complicações \\
\hline 9 & $\mathrm{C}$ & TIPO III-A & B3 & 30 & $P$ & Enc. $4 \mathrm{~cm}$ \\
\hline 25 & $\mathrm{C}$ & TIPO I & $\mathrm{C} 1$ & 30 & $\stackrel{\mathrm{H}}{\mathrm{N}}$ & Enc. $2 \mathrm{~cm}$ \\
\hline 37 & $\mathrm{C}$ & TIPO III-A & C3 & 11 & $\mathrm{H}$ & Enc. $4 \mathrm{~cm}$ \\
\hline 16 & PAF & TIPO I & B3 & 10 & $P$ & $10^{\circ}$ varo \\
\hline 22 & $\mathrm{C}$ & TIPO I & A3 & 7 & $\stackrel{\mathrm{H}}{\mathrm{N}}$ & $10^{\circ}$ valgo \\
\hline 18 & PAF & TIPO I & B3 & 18 & $P$ & IS.+ RC.+ RA. \\
\hline 33 & C & TIPO I & B2 & 10 & $\mathrm{H}$ & IS. + RA. \\
\hline 39 & C & TIPO I & $\mathrm{B} 2$ & 7 & $\stackrel{\mathrm{H}}{\mathrm{N}}$ & IS.+ RA. \\
\hline 27 & C & TIPO II & $\mathrm{B} 2$ & 10 & $\mathrm{H}$ & $\mathrm{FC} .+\mathrm{Fl}$ \\
\hline 6 & $\mathrm{C}$ & TIPO II & B3 & 1 & $P$ & FC.+ IP. \\
\hline 24 & C & TIPO II & B2 & 27 & $\mathrm{H}$ & IP. \\
\hline 38 & C & TIPO II & B2 & 20 & $\stackrel{\mathrm{H}}{\mathrm{N}}$ & IP. \\
\hline
\end{tabular}

Mec lesão = Mecanismo da lesão $;$ Class.Gustilo = Classificação de Gustilo $/ ;$ Class. AO = Classificação da AO $;$ T.espera = Tempo de espera $/$ Sint. $=$ Síntese $/ ; C=$ contusa $/ ;$ PAF = projétil de arma de fogo $/ ; \mathrm{P}=$ placa $/ ; \mathrm{H}=$ haste $/ ; \mathrm{Enc}=$ encurtamento $/ ; \mathrm{IS}=$ Infecção superficial $/ ; \mathrm{IP}=$ Infecção profunda $; \mathrm{RC} .=$ retarde de consolidação $; \mathrm{RA} .=$ Rigidez articular $/ \mathrm{FC}=\mathrm{Falha}$ de consolidação / . = Falha do implante

Tabela 3 - Distribuição dos pacientes de acordo com o mecanismo da lesão, classificações, tempo de espera, tipo de síntese e complicações. 
tados com hastes ${ }^{(11,13)}$. Como empregado por um grande número de cirurgiões, as hastes aqui utilizadas foram fresadas, porém, a inserção da haste foi a foco aberto. A aplicação da haste a foco aberto, a utilização de fresagem e o uso de haste canulada são fatores que influenciam o resultado nas fraturas expostas comparativamente à aplicada a foco fechado, sem fresagem e o uso de haste sólida ${ }^{(7,19)}$. Outra causa que pode estar associada ao alto índice de complicações é o fato destes pacientes permanecerem um tempo relativamente longo em tração, a espera do tratamento definitivo. Esses fatores associados a heterogeneidade de atendimento inicial para a limpeza de urgência realizada por diferentes cirurgiões ortopédicos e a curva de aprendizado para a aplicação das hastes, poderiam explicar nossos resultados.

Os resultados baseados na classificação de Thorensen, na análise global mostraram $75,7 \%$ de excelentes e bons resultados e na avaliação dos grupos, $85 \%$ de resultados excelentes e bons para o grupo das placas, e 75\% para o grupo das hastes, índices similares à literatura na avaliação de séries de fraturas fechadas com alguns casos de fraturas expostas ${ }^{(8,16,20,21)}$. (Figuras. 3 e 4 ).

A ausência de resultado estatisticamente significativo em favor do uso de placas em nosso trabalho, a conhecida superioridade biomecânica da haste embasada na literatura mundial, aponta para reforçar a abordagem com as hastes intramedulares sólidas bloqueadas aplicadas no mesmo ato do desbridamento quando possível ${ }^{(1,4,13)}$. Em alguns casos de fraturas expostas da diáfise do fêmur tipo IIIB e nos pacientes com comprometimento da função pulmonar, a haste deve ser usada com cautela ${ }^{(11)}$.

O segmento pós-operatório foi curto e novos ensaios devem ser realizados sem a inclusão das lesões penetrantes com amostras maiores e mais uniformes e segmento mais longo.

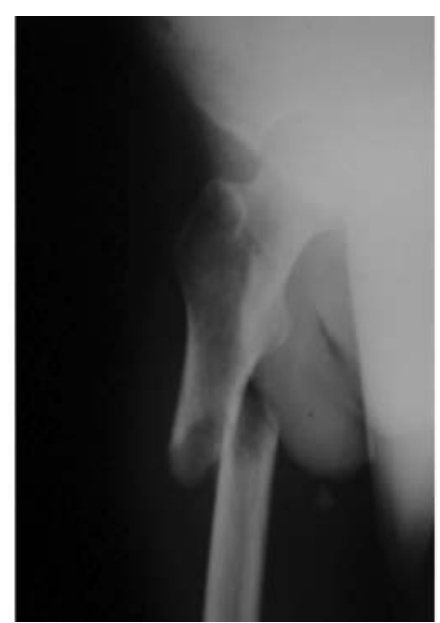

Figura 3 - 18 anos, masc, vítima de acidente motociclístico, com fratura exposta de fêmur $D$ classificada de Gustilo tipo I, AO- A3.

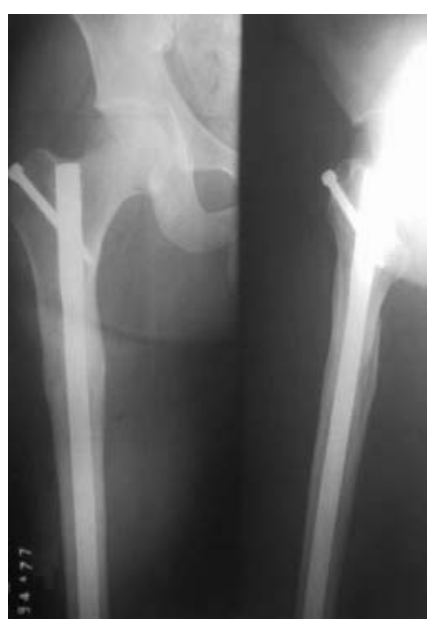

Figura 4 - Consolidou em 18,8 semanas e não houve complicação. 0 resultado pela avaliação de Thoresen foi excelente.

\section{CONCLUSÃO}

Lesões por trauma contuso e espera prolongada para a osteossíntese estiveram relacionados com um índice maior de complicações.

A técnica de estabilização com haste bloqueada intramedular fresada e bloqueada a foco aberto apresentou maior incidência de complicações quando comparada a osteossíntese com placas,embora sem significância estatística .

Nos resultados, pela avaliação de Thoresen, a definição não foi estatisticamente significativa, embora o tratamento com placa resultou em um índice maior de resultados excelentes e bons.

\section{REFERÊNCIAS}

1. O'Brien PJ, Meek RN, Powell JN, Blachut PA. Primary intramedullary nailing of open femoral shaft fractures. J Trauma. 1991; 31:113-6.

2. Hungria JS Neto. Fraturas diafisárias do fêmur. Ainda há indicação para o uso de placas ? Rev Bras Ortop. 1996; 31:444-8

3. Grosse A, Christie J, Taglang G, Court-Brown C, McQueen M. Open adult femoral shaft fracture treated by early intramedullary nailing. J Bone Join Surg Br. 1993; 75:562-5.

4. Van den Bossche MRP, Broos PL, Rommens PM. Open fractures of the femoral shaft, treated with osteosynthesis or temporary external fixation. Injury. 1995; 26:323-5.

5. Gustilo RB, Mendoza RM, Williams DN. Problems in the management of type III (severe) open fractures: a new classification of type III open fractures. J Trauma. 1984: 24:742-6.

6. Müller ME. The comprehensive classification of fractures of long bones. In: Müller ME, Allgöwer M, Schneider R, Willeneger H. Manual of internal fixation. 3th ed. Berlin: Springer-Verlag; 1992. p.118-50.

7. McLaren AC, Roth $\mathrm{JH}$, Wright $\mathrm{C}$. Intramedullary rod fixation of femoral shaft fractures: comparison of open and closed insertion techniques. Can J Surg. 1990; 33:286-90.

8. Thoresen BO, Alho A, Ekeland A, Stromsoe K, Folleras G, Haukebo A. Interlocking intramedullary nailing in femoral shaft fractures. J Bone Joint Surg Am. 1985; 67:1313-20.

9. Green A, Trafton PG. Early complications in the management of open femur fractures: a retrospective study. J Orthop Trauma. 1991; 5:51-6.

10. Noumi T, Yokoyama K, Ohtsuka H, Nakamura K, Itoman M. Intramedullary nailing for open fractures of the femoral shaft:evaluation of contributing factors on deep infection and nonunion using multivariate analysis. Injury. 2005; 36:1085-93

11. Williams MM, Askins V, Hinkes EW, Zych GA. Primary reamed intrame dullary nailing of open femoral shaft fractures. Clin Orthop Relat Res. 1995; (318): 182-90.

12. Long WT, Chang W, Brien EW. Grading system for gunshot injuries to the femoral diaphysis in civilians. Clin Orthop Relat Res. 2003; (408):92-100.

13. Tornetta $P$ 3rd, Tiburzi D. Reamed versus nonreamed anterograde femoral nailing. J Orthop Trauma. 2000; 14:15-9.

14. Bone LB, Johnson KD, Weigelt J, Scheinberg R. Early versus delayed stabilization of femoral fractures. J Bone Joint Surg Am. 1989; 71:336-40.

15. Baixauli F, Baixauli EJ, Sánchez-Alepuz E, Baixauli F Jr. Interlocked intramedullary nailing for treatment of open femoral shaft fractures. Clin Orthop Relat Res. 1998; (350):67-73.

16. Fernandes HJA. Placa em ponte e haste intramedular bloqueada: estudo comparativo no tratamento das fraturas multifragmentárias da diáfise do fêmur [tese]. São Paulo: Universidade Federal de São Paulo; 2000.

17. Riemer BL, Foglesong ME, Miranda MA. Femoral plating. Orthop Clin North Am. 1994; 25:625- 33

18. Böstman O, Varjonen L, Vainionpää S, Majola A, Rokkanen P. Incidence of local complications after intramedullary nailing and after plate fixation of femoral shaft fractures. J Trauma. 1989; 29:639-45.

19. Melcher GA, Hauke C, Metzdorf A, Perren SM, Printzen G, Schlegel U, et al. Infection after intramedullary nailing: an experimental investigation on rabbits. Injury. 1996; 27(Suppl):23-6.

20. Van Niekerk JLM, Schoots FJ. Femoral shaft fractures treated with plate fixation and interlocked nailing. A comparative retrospective study. Injury. 1992; 23:219-22.

21. Vasconcelos JW, Morais SV, Porto LCK, Santos RJM. Tratamento das fraturas cominutivas do fêmur com a utilização da placa em ponte. Rev Bras Ortop. 2004; 39:415-22 